\title{
Historical Developments of BHR Humanoid Robots
}

\section{Qiang Huang1,2, Zhangguo Yu ${ }^{1,2}$, Xuechao Chen1,2, Weimin Zhang1,2, Tianqi Yang1, Wenxi Liao1, Macro Ceccarelli 2,3}

\author{
${ }^{1}$ Intelligent Robotics Institute, School of Mechatronical Engineering, Beijing Institute of Technology, Beijing, China \\ ${ }^{2}$ Beijing Advanced Innovation Center for Intelligent Robots and Systems, Beijing Institute of Technology, Beijing, China \\ ${ }^{3}$ Department of Industrial Engineering, University of Rome Tor Vergata, Roma, Italy \\ Email: qhuang@bit.edu.cn, marco.ceccarelli@unicas.it
}

How to cite this paper: Huang, Q., Yu, Z. G., Chen, X. C., Zhang, W. M., Yang, T. Q., Liao, W. X., \& Ceccarelli, M. (2019). Historical Developments of BHR Humanoid Robots. Advances in Historical Studies, 8 , 79-90.

https://doi.org/10.4236/ahs.2019.81005

Received: January 20, 2019

Accepted: March 17, 2019

Published: March 20, 2019

Copyright $\odot 2019$ by author(s) and Scientific Research Publishing Inc. This work is licensed under the Creative Commons Attribution International License (CC BY 4.0).

http://creativecommons.org/licenses/by/4.0/

\begin{abstract}
Humanoid robots can achieve increasingly complex functions and adapt to more complex environments. To boost the development of humanoid robot technology, a team at Beijing Institute of Technology initiated the research on humanoid robots from 2000. Their research primarily focuses on stable walking, whole-body complex motion, human-robot interaction, and multimodal motion of humanoid robots. Thus far, the team has developed 6 generations of humanoid robots. The latest humanoid robot, BHR-6P, can achieve multi-mode motions (for example, walk, jump, fall protection, crawl and roll), which will significantly improve the ability of robot to adapt to the environment. This paper presented the historical evolution of BHR humanoid robots and outlined their functions and features.
\end{abstract}

\section{Keywords}

Humanoid Robots, Historical Developments, BHR Robots

\section{Introduction}

Humanoid robots are expected to achieve most human motions, adapt to complex environments and accomplish human-like tasks due to their similar shape to human body. The earliest full-scale humanoid robot is the "WABOT-1" developed in Waseda University, Japan (Lim \& Takanishi, 2007). For decades since then, some famous humanoid robots have developed, such as P2 (Hirai et al., 1998), WABIAN (Lim \& Takanishi, 2007), ASIMO (Hirose \& Ogawa, 2007), HRP (Kaneko et al., 2004), Johnnie (Löffler et al., 2004), HUBO (Cho et al., 2009), ATLAS (https://www.bostondynamics.com/atlas), and CASSIE 
(http://www.agilityrobotics.com/). In the 1980s, China began to study humanoid robots. Several teams from Harbin Institute of Technology, National Defense University of Science and Technology, Beijing Institute of Technology (BIT), Tsinghua University, and other universities, were formed for the humanoid robot research.

BIT launched the research of humanoid robots and formed a research team in 2000. The team leader is Prof. Qiang Huang. The team unveiled the BHR-1 humanoid robot in 2002 (Huang et al., 2002). The term BHR is the acronym from Beijing Institute of Technology Humanoid Robots. To promote the research of humanoid robot, the Intelligent Robotic Institute (IRI), BIT, was established with the team as the core in 2005. The director of IRI is also Prof. Qiang Huang. Up to now, the team in IRI, BIT, has developed 6 generations of BHR humanoid robots, from BHR-1 to BHR-6 (Huang et al., 2002; Huang et al., 2007; Yu et al., 2014b; Meng et al., 2015).

In general, from BHR-1 to BHR-5, the team focused on humanoid walking and manipulation (Chen et al., 2017; Jiang et al., 2018; Huang et al., 2018). The humanoid robots can walk, play the traditional martial arts (e.g. TaiChiand broadsword), perform human-like facial expressions, and play table-tennis. In order to have the humanoid robot adapt to complex unknown environments, from 2011, the team shifted their focus to enhance the environmental adaptabilities of humanoid robots via multi-mode motions (e.g. walk, jump, crawl, roll, climb, and fall protection). In 2017, BHR-6 with some basic multi-mode motions was unveiled. Despite significant progress in humanoid research, there is still a long way to break through technical challenges to go to bring humanoid robots to practical applications of serving humanity.

\section{Roadmap and Outline of BHR Humanoid Robots}

The BIT team of humanoid research strives to break through motion control methods, key components, and system integration of humanoid robots. Thus far, 6 generations of BHR humanoid robots have been developed by the team (Figure 1).
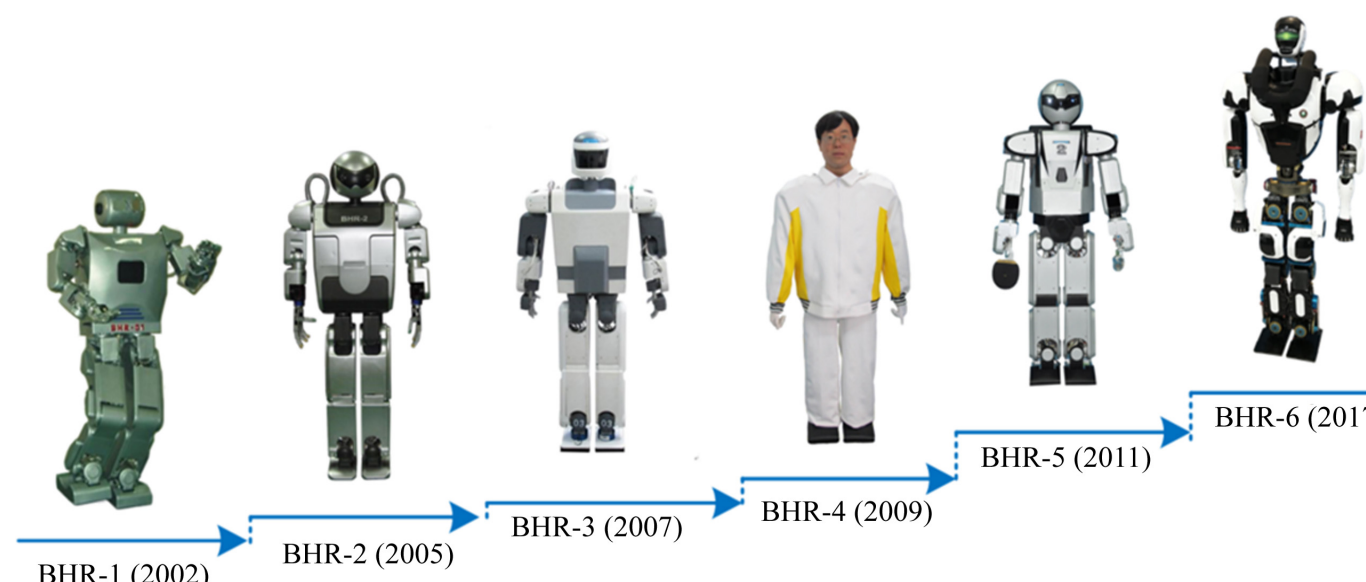

BHR-5 (2011) BHR-6 (2017)

BHR-1 (2002)

BHR-3 (2007)

BHR-4 (2009)

Figure 1. BHR humanoid robots from BIT. 
The BHR-1, the first generation of BHR humanoid robot, was unveiled in 2002. It is the first full-scale bipedal robot without external tether in China. It can dynamically walk. Based on BHR-1, BHR-2 unveiled in 2005 can perform whole body motion, such as tai-chi and some other Chinese martial arts. The third generation, BHR-3 unveiled in 2007, was designed for commercial use. The team enhanced its reliability, and sold some BHR-3 robots to several museums, for example, China Science and Technology Museum, and Guangdong Science and Technology Center. Then, the BHR-4 robot was unveiled in 2009 and it can mimic typical human expressions, such as smile, angry, surprise, sadness, and so on. The BHR-5 robot unveiled in 2011, as a testbed to study fast humanoid manipulation based on vision, can play table-tennis against human or robotic opponents. Two BHR-5 robots can rally up to 200 rounds. The latest generation, BHR-6 unveiled in 2017, has the capability of multi-mode motion, such as walk, crawl, roll, and fall protection and recovery.

\section{Development of the BHR Series}

\subsection{BHR-1-The First Full-Scale Bipedal Robot without Tether in China}

In 2000, the BIT team got budget for humanoid robot research from the State High-Tech Development Plan (863 Program) of the Ministry of Science and Technology of China and National Natural Science Foundation of China (NSFC). Two years later, the first generation of BHR series robot, BHR-1 was unveiled, which is the first fully integrated humanoid robot capable of dynamic walking without tether in 2002. It marks a significant progress in the field of humanoid robots in China. BHR-1 is $1.58 \mathrm{~m}$ tall, weighs $76 \mathrm{~kg}$ and has 28 degrees of freedom. It was equipped with gyro and acceleration sensors in the chest, force-torque sensor on each foot, and vision system. It was a testbed for bipedal walking and whole-body motion. The maximum walking speed of BHR-1 was $2 \mathrm{~km} / \mathrm{h}$. Some motion planning and balance control methods were validated on BHR-1 robots. At present, the BHR-1 robot is displayed in the History Museum of Beijing Institute of Technology (Figure 2).

\subsection{BHR-2-Achieving Complex Motion}

In 2005, BHR-2, the second generation of the BHR robot, was unveiled with the support from 863 Program and NSFC. BHR-2 robot is $1.6 \mathrm{~m}$ tall, weighs $63 \mathrm{~kg}$ and has 32 degrees of freedom. The BHR-2 robot improved the capability of complex motion control based on BHR-1. The BHR-2 can perform the traditional martial arts (Figure 3), such as Tai Chi and broadsword. BHR-2 also showed better balance control capability than BHR-1. In 2005, BHR-2 robots were invited to give live performance on CCTV-1 "Innovation China" TV program (Figure 4). The BHR-2 robots are selected as one of major national scientific and technological achievements of 863 Program of tenth Five-Year Plan and invited to participate in the Major Science and Technology Achievement Exhibition of 


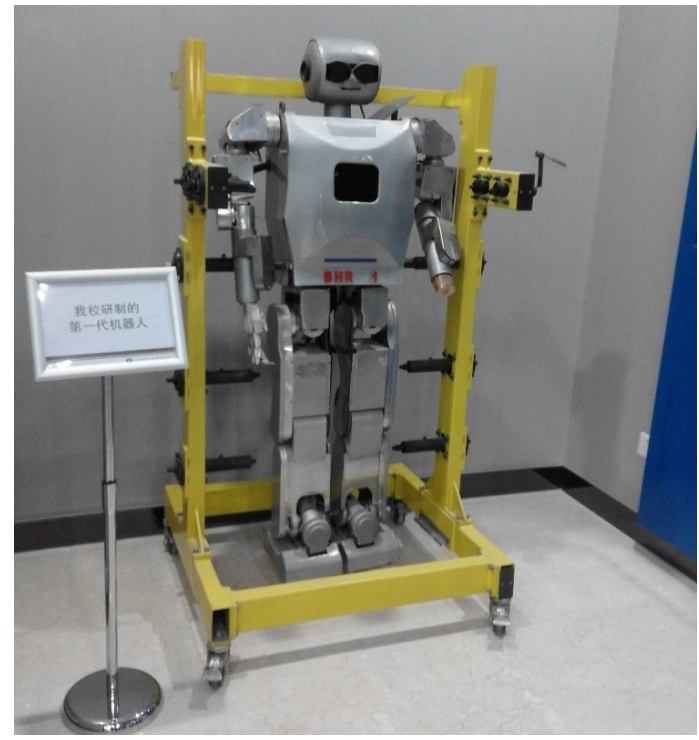

Figure 2. BHR-1 exhibited at BIT Museum in Beijing.

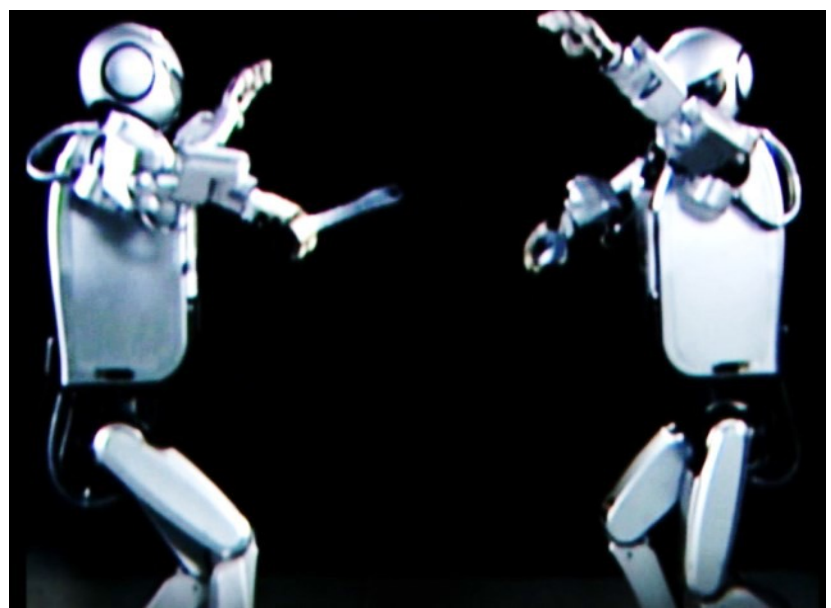

Figure 3. BHR2 performing martial arts.

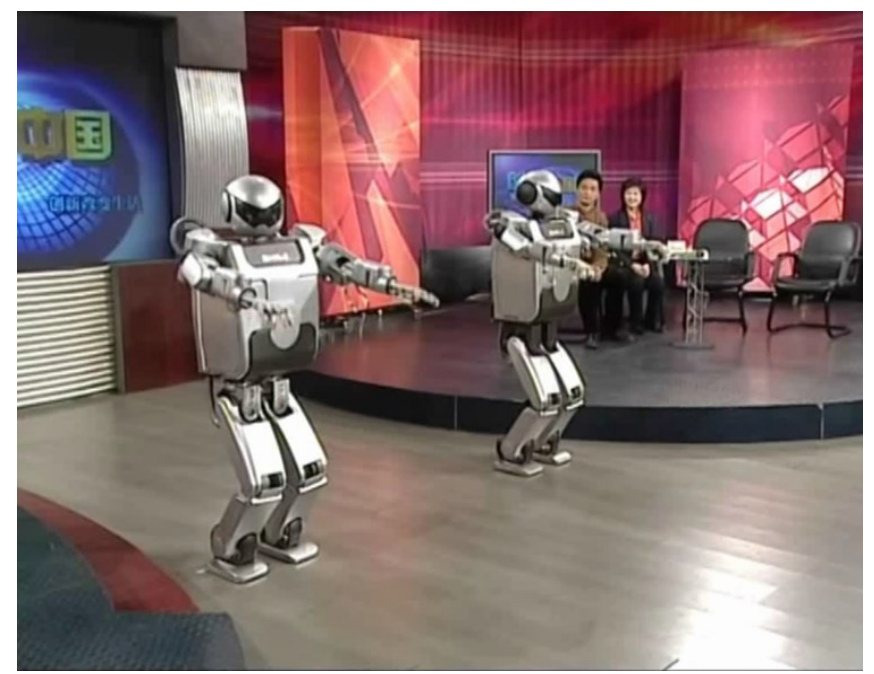

Figure 4. Live performance of BHR-5 robots on CCTV-1 in 2005. 
National Tenth Five-Year Plan of China in 2005. Many media reported the progress of BHR-2 robots.

\subsection{BHR-3-Commercial Humanoid Robot}

The third generation, BHR-3 unveiled in 2007, was designed for museums, for example, China Science and Technology Museum (Figure 5), Guangdong Science and Technology Center and Zhejiang Science and Technology Museum. It was the first time in China that adult-sized humanoid robots were sold as commercial product. BHR-3 was implemented with modular design, which significantly improved the reliability and efficiency of robot. The robots performed twice every day in museums.

\subsection{BHR-4-Humanoid Robot for Human-Robot Interaction}

The issue of human-robot interaction is important for having humanoid robots serve humans in daily life. A humanoid robot preferably has a humanlike face and makes facial expressions similar to human. In order to achieve this goal, the BIT team developed BHR-4 robot (Figure 6), which was unveiled in 2009. BHR-4 is very similar to the original model, a professor in the BIT team. It is 1.7 $\mathrm{m}$ tall, weights $65 \mathrm{~kg}$, and has 46 degrees of freedom. There are 13 active degrees of freedom in the robotic head. BHR-4 is capable of performing human-like facial expressions to express several typical emotions, such as happiness, anger, disgust, fear, sadness, and surprise (Yu et al., 2014a). Besides performing facial expressions, BHR-4 also has the functions of its previous generations, such as locomotion with two legs.

The BHR-4 was selected as a significant achievement and was exhibited on the $20^{\text {th }}$ Anniversary Achievement Exhibition of National High-tech Zone Construction in 2012. Many news media reported the research result (Figure 7).

\subsection{BHR-5 Robot-A Robot that Can Play Table Tennis}

Humanoid robots should not only be able to walk, but also have the ability of manipulation. In order to investigate humanoid manipulation, the BIT team began to develop a new humanoid robot in 2008. The fifth generation of the BHR robot, BHR-5, unveiled in 2011 can fulfill fast manipulation based on vision, such as playing table tennis against human and robotic opponents (Figure 8). Two BHR-5 robot played more than 200 rounds.

In order to play table tennis, BHR-5 first detects initial ball trajectory by stereo vision system at a speed of $125 \mathrm{~Hz}$, then predict the ball's subsequent trajectory by aerodynamics and bouncing model between the ball and the table, then plan whole body's motion for hitting the ball to desired landing area, and finally control the robot to accomplish the planned motion. To play table tennis, visual precision, movement speed and balance capability are strict requirements on the robot. If a humanoid robot can play table tennis, it would accomplish many other dynamic tasks. 


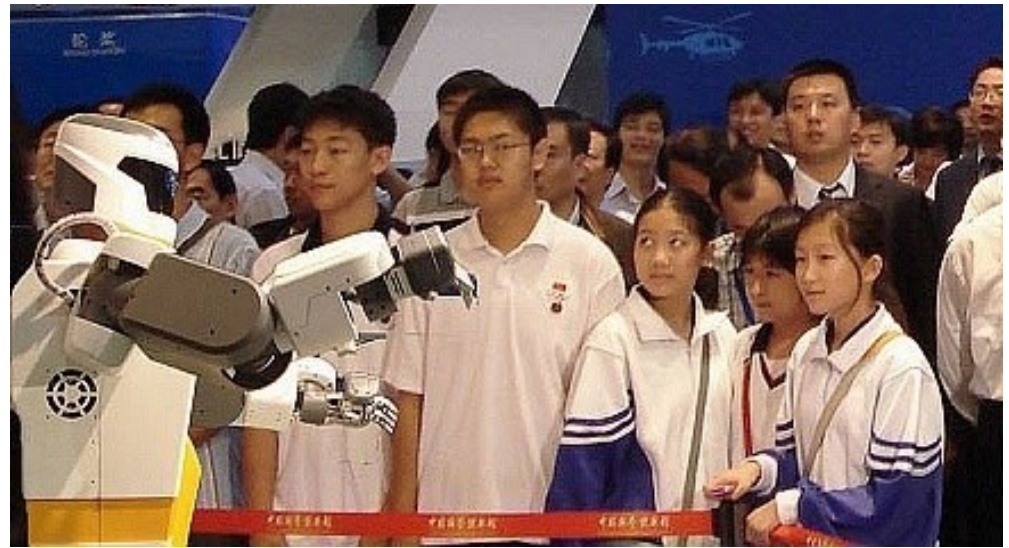

Figure 5. BHR-3 made demonstration in China Science and Technology Museum in Beijing.

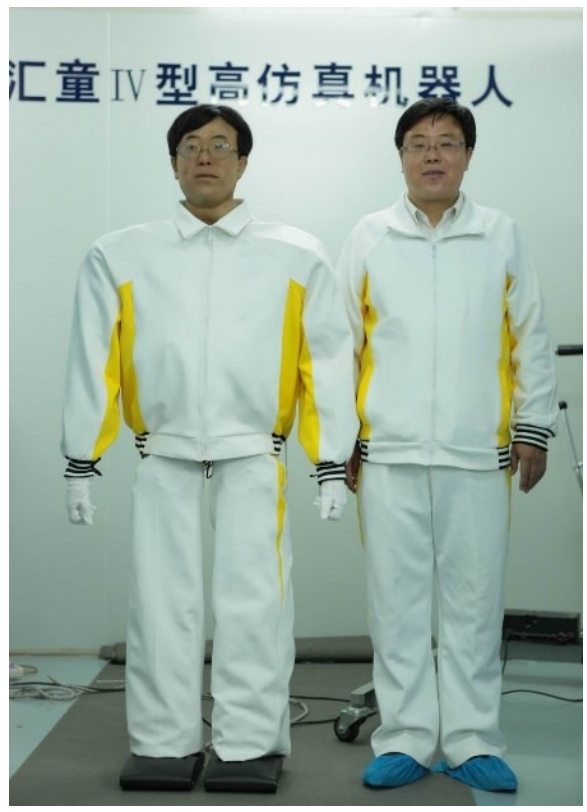

Figure 6. BHR4 and its human template.

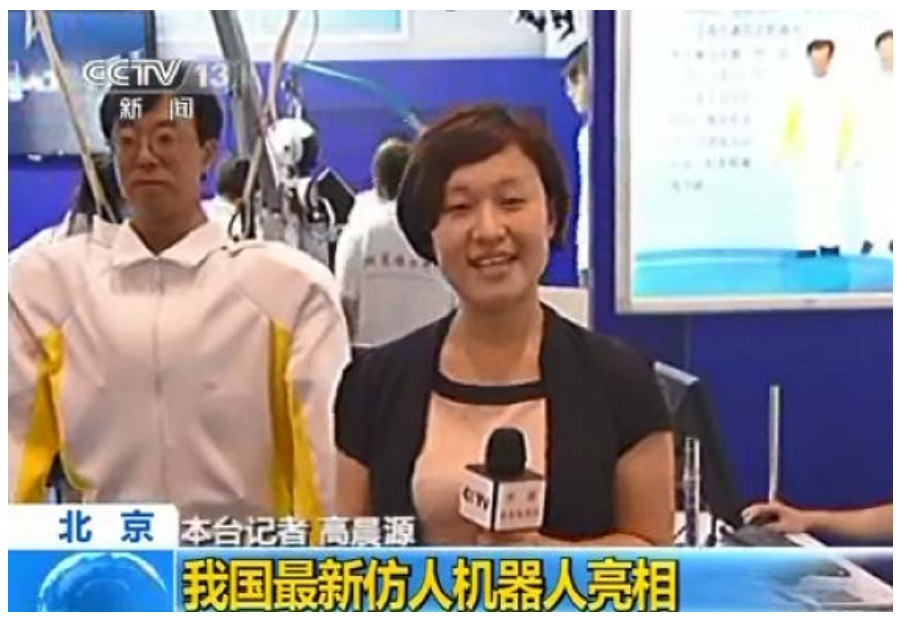

Figure 7. CCTV reported BHR-4 robot. 


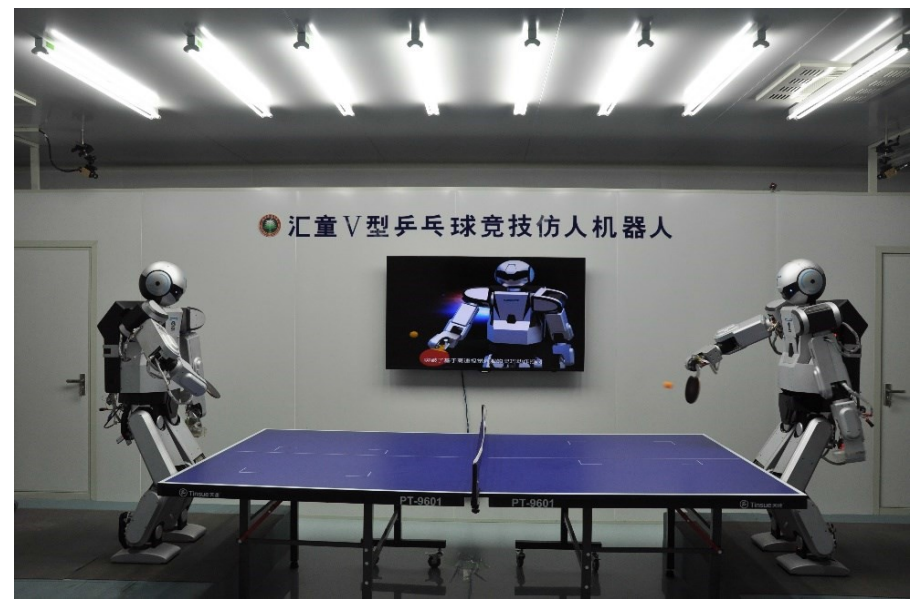

Figure 8. BHR-5 playing ping-pong in 2012.

The BHR-5 was also exhibited on the $20^{\text {th }}$ Anniversary Achievement Exhibition of National High-tech Zone Construction in 2012 (Figure 9 and Figure 10). The news media praised BHR-5 as one of highlights of the exhibition.

\subsection{BHR-6P Robot-Capable of Multi-Mode Motion}

Humanoid robots are expected to assistor replace humans to accomplish tasks in dangerous environments, for examples emergency rescue scenarios. To our experiences on humanoid experiments, although we try our best to keep a humanoid robot balance, the robot might fall down due to unexpected disturbance. It is almost inevitable to avoid falling in real environments. If a humanoid robot suffers from unexpected falling, the damage will be severe and the robot can't recover and continue to work. To grant a humanoid robot with multi-mode locomotion capability is a feasible way to make humanoid adapt to real environment. For example, if a robot suffers from falling down unexpectedly, it can try to protect itself and recover again. If a robot can't pass through low and narrow space by walking, it might choose crawling. If a robot can't pass through by crawling, it might select jumping.

To break through these problems of humanoid robot, the development of BHR-6 was launched in 2013. We conducted joint research with Takanishi Laboratory of Waseda University with the support of NSFC. In order to achieve multi-mode locomotion, the robot should have large range of movement. High strength and light weight mechanism are very critical for the robot. To endure large impact during falling and achieve large range of movement, a parallel differential mechanism consisting of two leadscrew drives was used in waist joints. In order to buffer and absorb impact during falling, some passive buffering material is attached on chest, knees, back, and hips.

In 2017, BHR-6 was unveiled, with the height of $1.65 \mathrm{~m}$, the weight of $55 \mathrm{~kg}$, and 23 degrees of freedom. BHR- 6 can walk on outdoor road, crawl, roll and take fall protection (Figure 11 and Figure 12).

BHR-6was exhibited in 2017 in the State Exhibition of Five Years of Great 


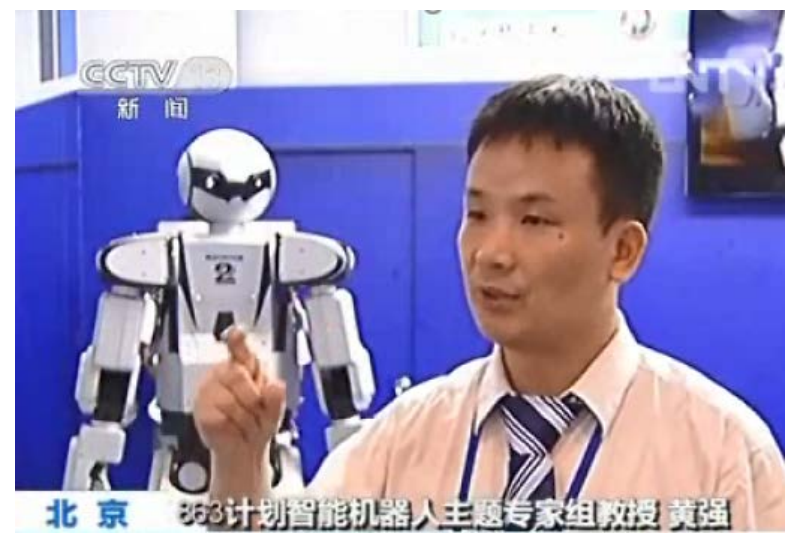

Figure 9. CCTV reported BHR-5 robot.

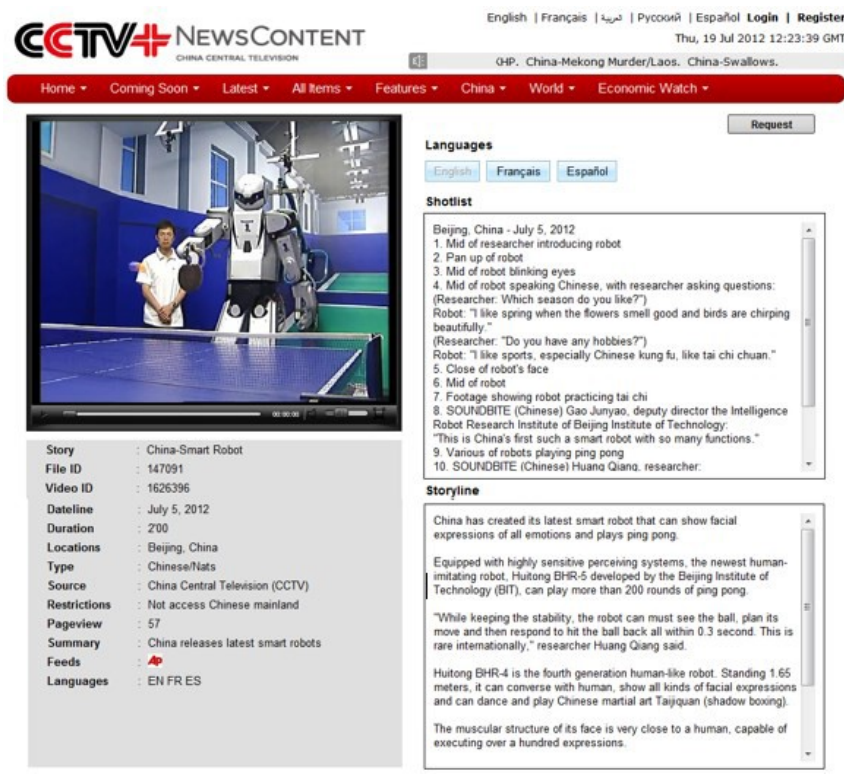

Figure 10. BHR-5 was reported by CCTV on Internet.

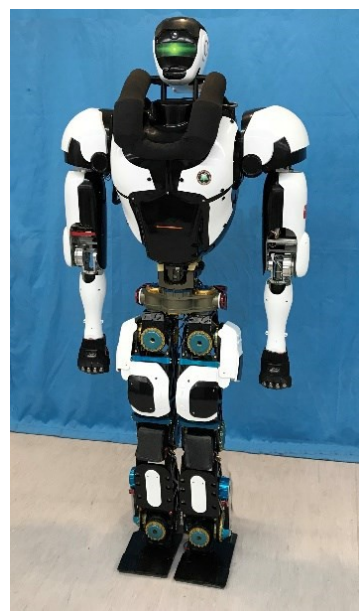

(a)

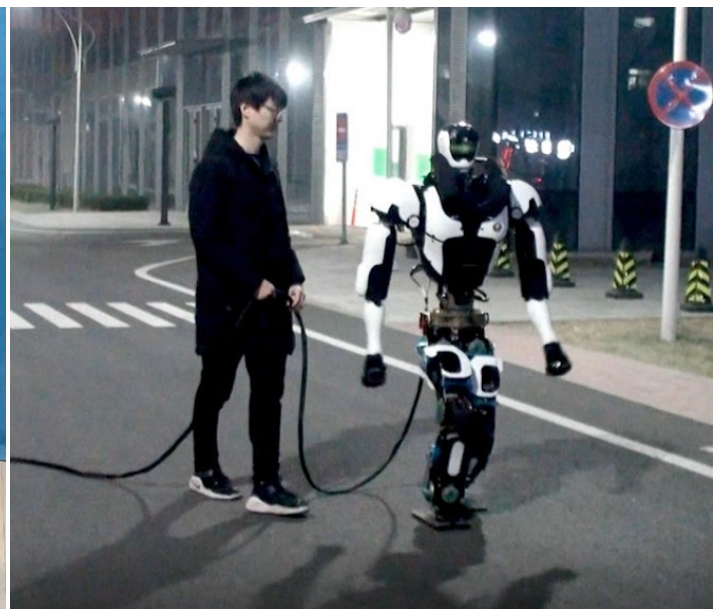

(b)

Figure 11. BHR-6 robot in 2017. (a) BHR-6 standing still; (b) BHR-6 walking on outdoor road. 
Achievements (Figure 13). In August 2018, the BHR-6 was exhibited in the exhibition of 2018 World Robotics Conference (Figure 14).

The 2018 IEEE-RAS 18th International Conference on Humanoid Robots (Humanoids 2018) took place in Beijing, China, on November 6 - 9, 2018. BIT hosted the conference and technical tour, and about 200 scholars witnessed the demonstration of BHR-6 humanoid robots during the technical tour (Figure 15).

All these significantly spread the impact of BHR humanoid robots at home and abroad.

\section{Future Work}

In the future, the BIT team will continue to focus the research on multi-mode motion of humanoid robot to enable BHR robots to have more motion modes and transform autonomously among different motions according to environmental conditions.

The BIT team of humanoid research continues to receive budget supports from

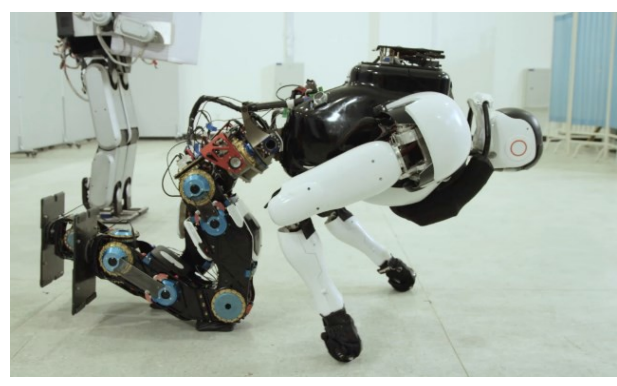

(a)

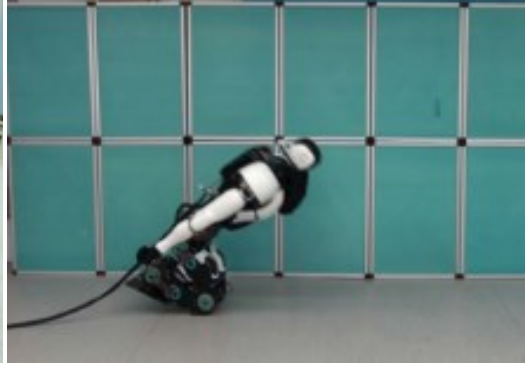

(b)

Figure 12. BHR-6 crawling and falling protection. (a) BHR-6 crawling; (b) BHR-6 kneeling to reduce impact while falling down.

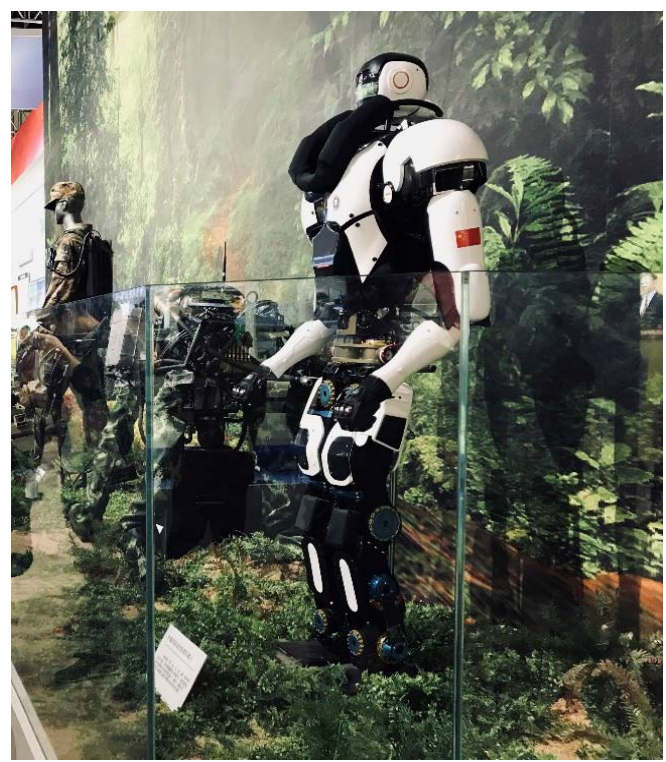

Figure 13. BHR-6P instate exhibition in 2017. 


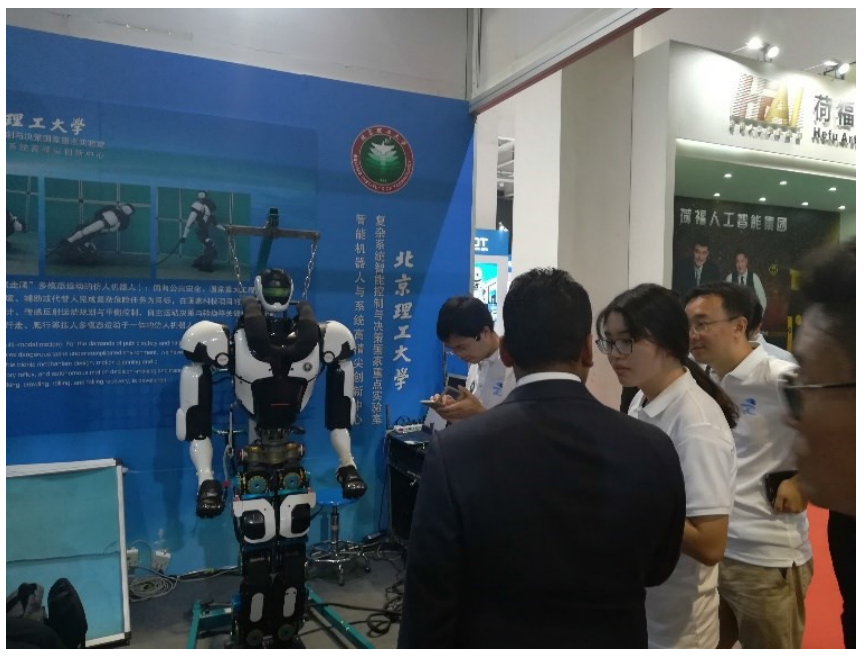

Figure 14. BHR-6P in the exhibition of 2018 World Robotics Conference.

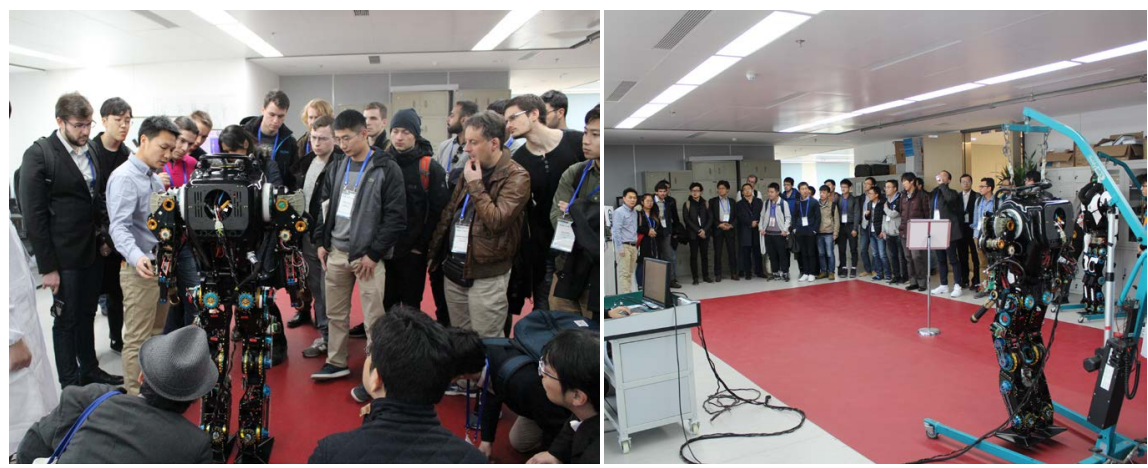

Figure 15. BHR-6P demonstration in Humanoids 2018 Conference.

NSFC, National Key R\&D Program of China, and other national technology plans. The team also gets strong support from Beijing Advanced Innovation Center for Intelligent Robots and Systems (BAICIRS), BIT.

The long-term goal of the BIT team is to develop humanoid robots that can work for the people at home or at danger, dirty, or dull environments instead of human.

\section{Conclusion}

In this paper, the development of six generations of BHR humanoid robots was outlined. Each generation of robots inherits the merits of the previous generation of robots and achieves further progress. At present, the BIT team pays more attention to enhancing the environmental adaptabilities of humanoid robots via multi-mode motions. The achievements of the BHR series have expanded the breadth and depth of the research on humanoid robots in China.

\section{Conflicts of Interest}

The authors declare no conflicts of interest regarding the publication of this paper. 


\section{References}

Chen, X. C., Yu, Z. G., Zhang, W. M., Zheng, Y., Huang, Q., \& Ming, A. G. (2017). Bioinspired Control of Walking with Toe-Off, Heel-Strike and Disturbance Rejection for a Biped Robot. IEEE Transactions on Industrial Electronics, 64, 7962-7971. https://doi.org/10.1109/TIE.2017.2698361

Cho, B.-K., Park, S.-S., \& Oh, J.-H. (2009). Controllers for Running in the Humanoid Robot, HUBO. Proceedings of the 9th IEEE-RAS International Conference on Humanoid Robots, Paris, 7-10 December 2009, 385-390.

Hirai, K., Hirose, M., Haikawa, Y., \& Takenaka, T. (1998). The Development of Honda Humanoid Robot. Proceedings of the IEEE International Conference on Robotics and Automation, 2, 1321-1326. https://doi.org/10.1109/ROBOT.1998.677288

Hirose, M., \& Ogawa, K. (2007). Honda Humanoid Robots Development. Philosophical Transactions of the Royal Society A: Mathematical, Physical and Engineering Sciences, 365, 11-19. https://doi.org/10.1098/rsta.2006.1917

Huang, Q., Li, K. J., \& Wang, T. M. (2002). Control and Mechanical Design of Humanoid Robot BHR-01. Proceeding of the Third IARP International Workshop on Humanoid and Human Friendly Robotics, 10-13.

Huang, Q., Yang, J., Yu, Z. G., Xu, W., Li, J. X., \& Li, K. J. (2007). Measurement of Human Walking and Generation of Humanoid Walking Pattern. 2007 IEEE International Conference on Robotics and Biomimetics (ROBIO), Sanya, 15-18 December 2007, 127-132. https://doi.org/10.1109/ROBIO.2007.4522147

Huang, Q., Yang, T. Q., Liao, W. X., Zhang, W. M., Yu, Z. G., Chen, X. C., \& Ceccarelli, M. (2018). Historical Development of BHR Humanoid Robots. In B. Zhang, \& M. Ceccarelli (Eds.), Explorations in the History and Heritage of Machines and Mechanisms. History of Mechanism and Machine Science (Vol. 37, pp. 310-323). Cham: Springer.

Jiang, X. Y., Chen, X. C., Yu, Z. G., Zhang, W. M., Meng, L. B., \& Huang, Q. (2018). Motion Planning for Bipedal Robot to Perform Jump Maneuver. Applied Science, 8, 139. https://doi.org/10.3390/app8010139

Kaneko, K., Kanehiro, F., Kajita, S. et al. (2004). Humanoid Robot HRP-2. Proceedings of the IEEE International Conference on Robotics and Automation, New Orleans, LA, 26 April-1 May 2004, 1083-1090. https://doi.org/10.1109/ROBOT.2004.1307969

Lim, H.-O., \& Takanishi, A. (2007). Biped Walking Robots Created at Waseda University: WL and WABIAN Family. Philosophical Transactions of the Royal Society A: Mathematical, Physical and Engineering Sciences, 365, 49-64. https://doi.org/10.1098/rsta.2006.1920

Löffler, K., Gienger, M., Pfeiffer, F., \& Ulbrich, H. (2004). Sensors and Control Concept of a Biped Robot. IEEE Transactions on Industrial Electronics, 51, 972-980. https://doi.org/10.1109/TIE.2004.834948

Meng, L. B., Yu, Z. G., Chen, X. C., Zhang, W. M., Ceccarelli, M., Hashimoto, K., Takanishi, A., Huang, Q., Guo, W. J., Xie, L., \& Liu, H. X. (2015). A Falling Motion Control of Humanoid Robots Based on Biomechanical Evaluation of Falling down of Humans. Proceedings of the 2015 IEEE-RAS 15th International Conference on Humanoid Robots (Humanoids), Seoul, 3-5 November 2015, 441-446. https://doi.org/10.1109/HUMANOIDS.2015.7363571

Yu, Z. G., Huang, Q., Ma, G., Chen, X. C., Zhang, W. M., Li, J., \& Gao, J. Y. (2014a). Design and Development of the Humanoid Robot BHR-5. Advances in Mechanical Engineering, 6, Article ID: 852937. https://doi.org/10.1155/2014/852937 
Yu, Z. G., Ma, G., \& Huang, Q. (2014b). Modeling and Design of a Humanoid Robotic Face Based on an Active Drive Points Model. Advanced Robotics, 28, 379-388. https://doi.org/10.1080/01691864.2013.867290

http://www.agilityrobotics.com/

https://www.bostondynamics.com/atlas 